\title{
BMJ Open Internet-based self-management support for adults with asthma: a qualitative study among patients, general practitioners and practice nurses on barriers to implementation
}

Johanna L van Gaalen, Leti van Bodegom-Vos, Moira J Bakker, Jiska B Snoeck-Stroband, Jacob K Sont

To cite: van Gaalen JL, van Bodegom-Vos L, Bakker MJ, et al. Internet-based selfmanagement support for adults with asthma: a qualitative study among patients, general practitioners and practice nurses on barriers to implementation. BMJ Open 2016;6:e010809. doi:10.1136/bmjopen-2015010809

- Prepublication history and additional material is available. To view please visit the journal (http://dx.doi.org/ 10.1136/bmjopen-2015010809).

Received 14 December 2015 Revised 30 June 2016 Accepted 29 July 2016

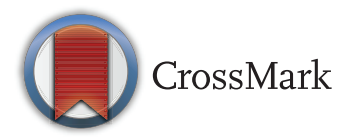

Department of Medical Decision Making, Leiden University Medical Centre, Leiden, The Netherlands

Correspondence to Dr Jacob K Sont; j.k.sont@lumc.nl

\section{ABSTRACT}

Objectives: The aim of this study was to explore barriers among patients, general practitioners (GPs) and practice nurses to implement internet-based selfmanagement support as provided by PatientCoach for asthma in primary care.

Setting: Primary care within South Holland, the Netherlands.

Participants: Twenty-two patients (12 women, mean age 38 years), 21 GPs ( 6 women, mean age 52 years) and 13 practice nurses (all women, mean age 41 years).

Design: A qualitative study using focus groups and interviews.

Outcomes: Barriers as perceived by patients, GPs and practice nurses to implementation of PatientCoach.

Methods: 10 focus groups and 12 interviews were held to collect data: 4 patient focus groups, 4 GP focus groups, 2 practice nurse focus group, 2 patient interviews, $5 \mathrm{GP}$ interviews and 5 practice nurse interviews. A prototype of PatientCoach that included modules for coaching, personalised information, asthma self-monitoring, medication treatment plan, feedback, e-consultations and a forum was demonstrated. A semistructured topic guide was used. Directed content analysis was used to analyse data. Reported barriers were classified according to a framework by Grol and Wensing.

Results: A variety of barriers emerged among all participant groups. Barriers identified among patients include a lack of a patient-professional partnership in using PatientCoach and a lack of perceived benefit in improving asthma symptoms. Barriers identified among GPs include a low sense of urgency towards asthma care and current work routines. Practice nurses identified a low level of structured asthma care and a lack of support by colleagues as barriers. Among all participant groups, insufficient ease of use of PatientCoach, lack of financial arrangements and patient characteristics such as a lack of asthma symptoms were reported as barriers.

Conclusions: We identified a variety of barriers to implementation of PatientCoach. An effective

\section{Strengths and limitations of this study}

- This study provides in-depth information on barriers to usage of internet-based self-management (IBSM) support as provided by PatientCoach among patients, general practitioners (GPs) and practice nurses. Our findings can be relevant for IBSM strategies in other chronic diseases.

- Our recruitment strategy was designed to include a diverse sample of patients and professionals.

- Our data have been obtained in one province in the Netherlands. The relevance and impact of our findings in other primary care settings are unknown.

- Participants have only been demonstrated a prototype of PatientCoach. Therefore, data are based on user expectations.

implementation strategy for internet-based selfmanagement support in asthma care should focus on these barriers.

\section{INTRODUCTION}

Asthma is characterised by variability in symptoms and airflow limitation. ${ }^{1}$ Therefore, asthma treatment should be adjusted over time. ${ }^{2}$ Self-management is an important aspect of the treatment to achieve and sustain asthma control. Self-management strategies consisting of self-monitoring, education, regular consultation with a professional and provision of an action plan have been demonstrated to improve health outcomes for patients with asthma. ${ }^{3}$ However, self-management strategies are poorly implemented within general practice. ${ }^{5-7}$ Internet technology might offer an attractive means for encouraging patients to use self-management strategies within a day-to-day context. ${ }^{8}$ This is demonstrated by the increasing number of available apps on 
asthma self-management. ${ }^{9}$ Previously, we developed internet-based self-management (IBSM) support for asthma, consisting of the following components: internet-based asthma monitoring, internet-based goal setting, decision support with a treatment plan, online medical review and tailored online information and communication with a healthcare provider. $^{10}$ IBSM support was based on focus groups, ${ }^{11}$ the Chronic Care model $^{12}$ and known key components for effective selfmanagement. ${ }^{3}$ The Chronic Care model is aimed at improving healthcare outcomes for patients with a chronic disease by means of a proactive patient-professional partnership by addressing organisational factors (ie, decision support systems) and resources (ie, selfmanagement support). It was developed to support patients in conducting self-management activities and to develop a patient-provider partnership in asthma care. ${ }^{13}$ Recently, we have shown that this IBSM support leads to improved asthma-related quality of life, asthma control and lung function as well as a greater number of symptom-free days as compared to usual care. ${ }^{10}$ Moreover, cost-effectiveness and long-term outcomes of this study showed that IBSM support is the preferred strategy as compared to current care in terms of a sustained improvement in quality of life with similar costs over a 1-year period. ${ }^{14}{ }^{15}$ Currently, we aim to implement this IBSM support within primary care. For the purpose of this study, we developed 'PatientCoach', which is based on our previous findings on IBSM support. It has been recommended that implementation strategies need to be tailored to factors either hampering ('barriers') or facilitating ('facilitators') take-up. ${ }^{16} 17$ Strategies that address barriers and facilitators at the patient, professional and organisational levels are the most successful in improving process and clinical outcomes. ${ }^{18}$ Therefore, the aim of this study is to explore and categorise all potential barriers associated with implementation of PatientCoach in asthma care within general practice as perceived by patients, practice nurses and general practitioners (GPs).

\section{METHODS}

\section{Design}

We conducted semistructured focus groups and interviews among patients, GPs and practice nurses. Interviews were held for those who were unwilling or unable to attend a focus group. Focus groups and interviews are effective methods for detecting obstacles to change within healthcare. ${ }^{19}$

\section{Setting}

In the Netherlands, a 'standard' general practice covers 2300 patients per GP. The Dutch guideline for general practice on asthma states that medical review should be performed at least once a year. ${ }^{20}$ This guideline is in concordance with the current international guidelines. ${ }^{1}$ In the Netherlands, all persons are required to have a healthcare insurance package, which covers primary care. During 2010, 90\% of the Dutch households had internet access and $\sim 80 \%$ had access to high speed internet. ${ }^{21}$ Our participant group was selected within the Leidenthe Hague region, which is located in South Holland, a province in the Netherlands with a high population density, containing urban and rural settings.

\section{Participant selection and recruitment}

We aimed to conduct three focus groups among patients, practice nurses and GPs, consisting of 6 to 8 participants per focus group. All participants were invited by using an information letter. We continued to send invitations until we included sufficient participants. For the purpose of this study, we aimed to include GPs and patients with and without experience with IBSM support guided by a respiratory nurse from the Leiden University Medical Centre (LUMC) via a website. Therefore, some of the patients and GPs were sought among the participants of the previously conducted Self-Management of Asthma Supported by Hospitals, ICT, Nurses and GPs (SMASHING) study. In this study, we demonstrated the cost-effectiveness of IBSM support. The SMASHING website included modules for selfmonitoring, education and contact with a professional. Full details of this study have been published elsewhere. $^{1015}$ This was in contrast to the current study, as PatientCoach has been developed for guidance of patients by their own GP and/or practice nurse.

First, we selected GPs. To include GPs who had previously participated in the SMASHING study, we invited GPs from the Leiden general practice network (LEON). Additionally, we invited non-LEON network GPs. In total, we invited 150 GPs by information letter; of whom, 27 responded positively to participate in focus groups/interviews. Twenty-one GPs participated (participation rate $14 \%)$. Reasons for not participating included no time $(\mathrm{n}=2)$, no show $(\mathrm{n}=1)$ and unknown $(\mathrm{n}=126)$. Positively responding GPs were asked permission to invite their patients and practice nurses to participate. Unfortunately, we were not able to directly invite patients who participated in the SMASHING study, as informed consent was not obtained to approach patients in future studies. Patient inclusion criteria were physician-diagnosed asthma, age 18-50 years, use of inhaled corticosteroids and/or montelukast for at least 3 months in the previous 2 years, access to internet, no serious comorbid conditions (ie, terminal illness or a severe psychiatric disease) and ability to understand Dutch. From 13 practices (1 general practice covered 2 separate practices), we randomly selected 10 patients (130 patients) per practice; of whom, 22 patients ultimately participated (participation rate 17\%). Reasons for declining to participate were no asthma symptoms $(n=6)$, lack of time $(\mathrm{n}=4)$, Ramadan $(\mathrm{n}=1)$ and unknown $(\mathrm{n}=108)$.

In total, we invited 27 practice nurses, of whom 24 responded positively and 13 ultimately participated (participation rate $48 \%$ ). Reasons for declining to participate 
were lack of time $(\mathrm{n}=1)$, lack of financial reimbursement $(\mathrm{n}=1)$ and unknown $(\mathrm{n}=9)$.

\section{IBSM support}

IBSM support consists of a generic web-based system and an instruction visit for patients. The current generic web-based system is called 'PatientCoach' (http://www. patientcoach.nl). PatientCoach supports selfmanagement of patients with a chronic condition (see online supplementary file 1 ). It includes modules for coaching, personalised information (ie, inhalation technique of medication), self-monitoring (ie, Asthma Control Questionnaire), reminders, medication treatment plan, (motivational) feedback, e-consultations and a forum. PatientCoach has been developed by the LUMC. During the duration of this study, only a prototype version of PatientCoach was available. Input of participants of this study has been used for further development of PatientCoach.

\section{Focus groups and interviews}

Focus groups and interviews were conducted in 2010. Focus groups were performed at the LUMC and were conducted separately for each participant group. Focus groups were not held separately for those who had previously participated in the SMASHING study. We used focus group procedures of Morgan et $a t^{22}$ to prepare and guide focus groups. Interviews were held at the LUMC, at the general practice or at the patient's home.

During focus groups and interviews, a topic guide was used (see online supplementary file 2). We explained the concept of self-management, background of IBSM support and demonstrated PatientCoach. Hereafter, GPs and practice nurses were asked how routine asthma care is currently organised, and how self-management is implemented. Patients were asked how their current asthma care is arranged, and how they felt about selfmanagement. All participants were asked to give positive and negative comments about PatientCoach and to identify what they would need to start using PatientCoach. To assess whether the content of our topic list required changes, we analysed data from the first three focus groups prior to further data collection. No major adjustments were deemed necessary on the basis of this analysis.

A trained moderator (JLvG) and an observer (LvB-V or MJB) conducted focus groups. JLvG is a qualified medical doctor and has received postgraduate training on conducting qualitative research. The moderator and observers had no involvement in patient care, and the participants had no personal background information on the interviewers. Focus groups lasted 1.5 hours. JLvG conducted interviews, which lasted $\sim 40 \mathrm{~min}$. Focus groups and interviews were conducted until data saturation was reached, that is, until no new barriers emerged in three consecutive focus groups or interviews for a given participant group. ${ }^{23}$ Focus groups and interviews were audio-taped and fully transcribed. All focus groups and interviews were held in Dutch.
In patients, asthma control was assessed using the Asthma Control Questionnaire. ${ }^{24}{ }^{25}$ Lung function was measured as forced expiratory volume in $1 \mathrm{~s}\left(\mathrm{FEV}_{1}\right)$ using a hand-held electronic spirometer (PiKo1: nSpire Health, Longmont, Colorado, USA).

\section{Data analysis}

Directed content analysis was used to analyse all focus groups and interviews. This method is well suited for research that extends conceptually to a framework. ${ }^{26} \mathrm{We}$ used the framework developed by Grol and Wensing. ${ }^{17}$ This framework categorises barriers and facilitators into six domains of healthcare, namely the innovation in this case PatientCoach (eg, ease of use), the individual professional (eg, willingness to change), the patient (eg, perceived benefit), the social context (eg, support by colleagues), the organisational context (eg, availability of professionals) and the economic and political context (eg, financial arrangements). This information can be used to develop a tailored strategy and to facilitate implementation of PatientCoach in routine asthma care. We used predetermined barriers of this framework. ${ }^{17}$ New categories were developed for those barriers that could not be categorised within these predetermined barriers. Transcripts were coded independently by two researchers (JLvG and MJB). Coding was compared and discrepancies were discussed until consensus was achieved. After coding, JLvG and MJB independently classified barriers in the appropriate domains of the framework. The first interviews and focus groups were discussed with the complete research team. Analyses were undertaken using the software NVivo V.10 (2012; QSR International). The results have been reported in accordance with the Consolidated Criteria for Reporting Qualitative Research (COREQ) checklist. ${ }^{27}$

\section{RESULTS}

\section{Characteristics of the population}

Four focus groups were held with patients $(n=20)$, four with GPs $(n=16)$ and two with practice nurses $(n=8)$. The average number of participants in each focus group is four. Interviews were conducted with two patients, five GPs and five practice nurses. Tables 1 and 2 summarise the characteristics of the patients and professionals who participated in the focus groups and interviews. The participating patients covered a range with respect to age and level of asthma control. The participating GPs and practice nurses covered a wide range with respect to age, years of experience and a variety in general practice settings. One GP and six patients had previously participated in the SMASHING study.

\section{Barriers to implementation of PatientCoach according to patients}

For greater clarity, we will describe all found factors as potential barriers for implementation of PatientCoach. We identified a variety of barriers as perceived by patients (box 1) and grouped them into 13 categories. All categories are illustrated by a representative remark. 
Table 1 Patient characteristics

\begin{tabular}{ll}
\hline & $\begin{array}{l}\mathbf{N}(\%) \\
(\mathbf{n = 2 2})\end{array}$ \\
\hline $\begin{array}{l}\text { Age (years), mean (range) } \\
\text { Gender }\end{array}$ & $38(20-51)$ \\
$\quad$ Female & 55 \\
ACQ* score, mean (range) & $1.2(0-2.9)$ \\
Prebronchodilator FEV , $_{1}$ predicted, range & $94(79-107)$ \\
Level of education & \\
$\quad$ Low & 45 \\
$\quad$ Unknown & 10 \\
$\quad$ High & 45 \\
Ethnicity & $22(100)$ \\
$\quad$ Dutch & \\
\hline All variables are in \% except where indicated. \\
Low education indicates that persons whose highest education \\
level is primary education, junior general secondary education or \\
lower vocational education. \\
*Asthma Control Questionnaire, range (0) optimal asthma control- \\
(6) uncontrolled asthma. \\
ACQ, asthma control questionnaire; FEV ${ }_{1}$, forced expiratory \\
volume in 1 s; PN, practice nurse.
\end{tabular}

Table 2 GP and practice nurse characteristics

\begin{tabular}{lcc} 
& GPs $(\mathbf{n = 2 1 )}$ & $\begin{array}{l}\text { Practice } \\
\text { nurses }(\mathbf{n = 1 3})\end{array}$ \\
\hline Females & 29 & 100 \\
Age (years), mean (range) & $52(36-60)$ & $41(27-58)$ \\
Years practising as a GP or PN & \\
5 & 0 & 54 \\
$5-10$ & 19 & 46 \\
$>10$ & 81 & 0 \\
Number of GPs working within general practice \\
$\leq 2$ & 52 & 31 \\
Setting & \multicolumn{2}{|c}{} \\
Urban & 57 & 62 \\
Rural & 43 & 38 \\
\hline All variables are in \% except where indicated. \\
ACQ, asthma control questionnaire; GP, general practitioners; PN, \\
practice nurse.
\end{tabular}

\section{Domain 1: characteristics of PatientCoach}

Almost all patients felt PatientCoach should be used within the context of a patient-professional partnership, as in contrast to using PatientCoach without guidance of a professional. Another item that was mentioned by almost all patients is insufficient ease of use: layout of the user interface should be straightforward and allow for tailoring to their individual needs, that is, by adjusting reminder settings for the frequency of monitoring asthma control. Other mentioned barriers included too much time investment and lack of security. Finally, patients identified a lack of evidence on PatientCoach content as a barrier.

\section{Domain 2: characteristics of the individual professional}

Our patients suggested that their decision to start using PatientCoach would not be influenced by which type of professional, either a GP or a practice nurse, would guide them. However, a lack of sufficient knowledge and skills of the professional on asthma management would influence their willingness to use PatientCoach.

\section{Domain 3: characteristics of the individual patient}

Some patients felt that PatientCoach is impersonal and, therefore, they would not be willing to use it. On being asked what patients would halt from using PatientCoach, most patients mentioned that a lack of potential benefit in terms of symptom reduction would be an important hampering factor. Patients related this to a level of current symptoms and subsequent willingness to change daily routines. Some patients stated that they did not perceive sufficient asthma symptoms or do not perceive asthma as a chronic condition, and are therefore not willing to routinely monitor their current level of asthma control. It is noteworthy to mention that the one patient who previously participated in the SMASHING study identified the gained insight in the actual level of asthma control as the main benefit of using IBSM support. Patients mentioned that PatientCoach might not be suitable for elderly people.

\section{Domain 4: characteristics of the organisational context}

During the focus groups, variation in the level of structured asthma care within general practices emerged as a theme. Sometimes, asthma care consisted only of obtaining a repeat prescription for maintenance medication. This is important as PatientCoach has been developed based on a proactive care approach, which requires regular assessment that allows for tailoring of treatment strategies to the individual patient needs.

\section{Domain 5: characteristics of the economic context}

Almost all patients mentioned that PatientCoach.nl should be free of user charge, including the lung function meter.

\section{Domain 6: characteristics of the social context}

No barriers emerged within this domain. Patients liked the functionality of a forum within PatientCoach to contact other patients.

\section{Barriers to implementation of PatientCoach according to professionals}

Among GPs and practice nurses, we identified barriers that we grouped into 18 categories. Box 2 presents transcripts of comments, grouped according to the six domains of the theoretical framework.

\section{Domain 1: characteristics of PatientCoach}

GPs and practice nurses mentioned that design and content should be straightforward and easy to integrate into the work routines of professionals. In the Netherlands, all general practices are required to use an electronic medical registry system. A lack of integration of PatientCoach within these systems is perceived as an important barrier to PatientCoach use among 
Box 1 Patients: barriers to PatientCoach usage, an overview of transcripts

Domain 1. Characteristics of PatientCoach

Lack of a patient-professional partnership

"The danger is that the GP is not involved. Maybe l'm old fashioned, but my GP has the knowledge and skills on asthma that can't be replaced." (Male 25 years)

Insufficient ease of use

"I don't want to monitor my symptoms weekly. That would be too much of a time investment." (Male, 29 years)

"You should not have to go through a complete website in order to gain insight in your actual level of asthma control." (Male, 39 years)

Time consuming

"It's (PatientCoach) a nice system. But I just lack time to use it." (Female, 48 years)

Lack of evidence

"l'm willing to use it (PatientCoach), unless it's not clear that a professional with sufficient knowledge has developed it." (Male, 20 years)

Lack of security

"This website contains personal data. This requires a very high level of protection." (Male, 20 years)

Domain 2. Characteristics of the individual professional

Lack of knowledge and skills on asthma management

"I often do experience that if you've told your complete story, the professional you're talking too replies with: I have to discuss this with someone else. That is annoying. It should be guaranteed that the professional who is guiding you should have sufficient knowledge and skills." (Female, 48 years)

Domain 3. Characteristics of the individual patient

Negative attitude towards PatientCoach

"I don't like it at all. I'm not interested in using the Internet. I believe that my GP should handle my asthma." (Female, 48 years)

Lack of outcome expectancy

"My asthma is OK now. I can imagine that PatientCoach could be useful if you are wondering how your asthma is doing, if you are wonder-

ing if you are doing the right thing. Then it makes sense. But now, it won't add anything as my asthma is OK." (Female, 51 years)

"I am afraid about self-confrontation. When you're doing well and start smoking and all your graphs show you're getting worse." (Male,

24 years).

Perception of asthma

"During the summer I usually stop taking my maintenance medication (flixotide), but I tend to wait too long to restart my medication. Since two weeks I'm feeling exhausted when I wake up_and now I'm thinking I should restart it." (Female, 37 years)

Difficulties changing routines

"I take my inhalers twice daily and (because of this) l'm doing well. l'm not willing to change this." (Male, 20 years).

"PatientCoach depends on self-discipline. I do believe that self-monitoring works, but this self-discipline for regular assessment of asthma control would be a barrier for me." (Female, 51 years)

Patient characteristics

"Maybe for elderly people, internet is too complicated, or elderly might not have access to the internet." (Female, 46 years)

Domain 4. Characteristics of the organisational context

Lack of routine asthma care

"I do not attend my general practice on a regular basis. Only when symptoms get worse." (Male, 30 years)

Domain 5. Characteristics of the economic context

User fee

"I am not willing to pay for using PatientCoach, or a lung function monitor. It should be covered by the insurance, as it leads to improved outcomes, and therefore cost reduction." (Male, 30 years)

Domain 6. Characteristics of the societal context

None.

professionals. Another emerging theme was that some professionals felt that PatientCoach is impersonal.

\section{Domain 2: individual professional}

A lack of a positive attitude towards PatientCoach was identified as a barrier among GPs and practice nurses to PatientCoach use. This attitude seems to be influenced by the perceived level of benefit and sense of urgency with respect to asthma care. For instance, GPs identified a lack of favourable outcomes of a cost-effectiveness analysis as a barrier. Moreover, GPs demonstrated differing senses of urgency towards asthma care. Among professionals working in practices without structured asthma care, a more passive approach towards asthma management was identified. This is in contrast to work routines of professionals in practices with structured asthma care, who vary professional involvement according to the needs of the individual patient, which correlates with the approach of self-management. Practice nurses working in practices without structured asthma care identified a lack of perceived self-efficacy as a barrier. Additionally, this level of perceived self-efficacy seems to be influenced by practice nurse characteristics, such as educational level. Those practice nurses with insufficient education reported to feel less confident in providing asthma care. 
Box 2 General practitioners (GPs) and practice nurses: barriers to PatientCoach usage, an overview of transcripts

Domain 1. Characteristics of PatientCoach

Insufficient ease of use

"What is most annoying is that this is not integrated within our electronic medical registry system. ... [..] I don't want to have to type in all lung function or asthma control measurements from this portal (PatientCoach) into this system." (GP, female, 43 years)

Time consuming

"The goal of PatientCoach to improve quality of asthma care. This does not have to imply a reduction in time investment. However, it should not require too much time investment." (GP, female, 47 years)

Lack of security

"Currently, I am using my email for patient contact. However, this sometimes involves personal information. That is secure. For PatientCoach I think this should be properly arranged." (Practice nurse, female, 39 years)

Domain 2. Characteristics of the individual professional

Negative attitude

"I prefer to see patients in real life. When they're entering my consultation room my observation starts-that's invaluable." (GP, male, 53 years)

Lack of perceived level of benefit

"If a patient is taking his/her medication on a regular basis, I wonder if internet-based self-management really results in improved outcomes.... [..] in terms of reduced number of exacerbations and in quality of life." (GP, male, 51 years)

Low sense of urgency with respect to asthma care

"I can't remember if I have had an emergency due to an asthma attack. Asthma is not that severe... apparently the self-management of patients is very good ... probably due to the improved efficacy of inhalation therapy." (GP, male, 61 years)

Current work routines

"I only see patients when they're having an exacerbation, or when I feel that someone is contacting too often for a refill of Ventolin." (GP, male, 57 years)

Lack of perceived self-efficacy

"It's important to have sufficient knowledge, to be able to explain your treatment advice to a patient. [..] The asthma protocol has to be written. Currently, I would refer patients to a GP as I don't have the knowledge and experience to guide asthma patients." (Practice nurse, female, 49 years)

Characteristics professional

"I am a qualified nurse. Luckily, I also received training in diabetes care and pulmonary medicine. It would be very unpractical if I had not received this training." (Practice nurse, female, 34 years)

Domain 3. Characteristics of the individual patient

Difficult target group

"Routine asthma care is difficult to organize. Patients do not attend their routine asthma consultations." (Practice nurse, female, 59 years)

"Patients often visit our practice too late, as they think their asthma is doing fine, when it's clearly not." (GP, male, 60 years)

Difficulties changing routines

"Asthma patients are difficult to motivate, both for attending routine consultations as for therapy adherence." (GP, male, 45 years)

Patient characteristics

"Patients do need certain skills in order to use the Internet. I think it's unsuitable for elderly or first generation immigrants." (GP, male, 53 years)

Characteristics asthma

"If asthma is under control, there's no sense in using it in terms of benefit." (GP, male, 58 years)

Domain 4. Characteristics of the organisational context

Lack of routine asthma care

"We do not have a protocol for asthma [..] Currently we are targeting diabetes, cardiovascular risk management in the elderly. Later on we will address COPD and asthma. COPD will be prioritized more highly." (Practice nurse, female, 55 years)

General practice characteristics

"Our practice is located in a rural setting. Our patients do not use the internet as often as those who are living in the city." (Practice nurse, female, 38 years)

Lack of availability of staff, tools and consultation rooms

"Nowadays, more sophisticated tools are available. Unfortunately I do not have them in my back pocket. For example a lung function meter.

These are the tools you're looking for that enable patients to monitor their symptoms." (GP, male, 57 years)

"If there's only one practice nurse, it's more difficult to guarantee continuity of care." (GP, female, 36 years)

Domain 5. Characteristics of the economic context

Lack of financial arrangements

"Financial arrangements are important. You need to be reimbursed for your consultation time. A regular control visit lasts 20 minutes, which is hardly enough time." (Practice nurse, female, 59 years)

Domain 6. Characteristics of the societal context

Lack of support by colleagues

"I find it hard to arrange routine asthma consultations within my practice; l'm just the only practice nurse." (Practice nurse, female, 35 years) 
Domain 3: individual patient

Practice nurses and GPs identified patients with asthma as a challenging target group: patients with asthma do often not attend their routine consultations and are often not adherent to their medication regimen. This was perceived as a barrier for PatientCoach use. PatientCoach was not found to be suitable for all patients with asthma, for example, for patients with a low level of symptoms, elderly patients or those who are illiterate or do have problems speaking and understanding Dutch.

\section{Domain 4: organisational context}

Practice nurses identified a low level of structured asthma care as a barrier. A low level of asthma care was often illustrated by a lack of protocol. This lack of structured asthma care was often attributed to a low sense of urgency towards asthma care within their general practice. Some professionals expressed that although they were enthusiastic about PatientCoach, their practice location in a rural setting or in a setting with immigrants would make it difficult to implement PatientCoach. To provide asthma care using PatientCoach, GPs identified that they would need the availability of sufficient equipment and staff.

\section{Domain 5: economic context}

Almost all professionals identified a lack of financial arrangements with insurance companies as an important factor relating to sustained PatientCoach usage.

\section{Domain 6: social context}

Another impeding factor mentioned by practice nurses was the lack of peer support from colleagues.

\section{DISCUSSION}

This study addresses a variety of barriers to the implementation of IBSM support as provided by PatientCoach, which we developed based on previous research on IBSM support in asthma. ${ }^{10}$ To the best of our knowledge, this is the first study that explores barriers among patient, practice nurses and GPs on IBSM support for asthma within primary care. We identified barriers at different domains of the theoretical framework by Grol and Wensing. ${ }^{17}$

First, at the domain of PatientCoach, patients and professionals identified usability issues that need to be addressed. For patients, this included sufficient functionalities to tailor PatientCoach settings to their individual needs, for instance, by adjusting monitoring frequency for measuring asthma control. For GPs, this included integration of PatientCoach within the electronic medical registry system. These findings resemble the current literature, in which screen data and context-related factors, like ability to work on a laptop or tablet, ${ }^{28}$ colour schemes ${ }^{29}$ and integration with software systems used by healthcare providers, have been reported to influence ease of use. ${ }^{30}$ Perceived ease of use is known to influence acceptance of new technology. ${ }^{31}$ It is noteworthy to mention the method of 'usercentred design', referring to actual involvement of end-users during the design process as a method for developing a health information system. ${ }^{32} 33$ Another important factor perceived by patients is the need for personal guidance in using PatientCoach. This need for personal guidance was found in studies involving other chronic diseases, like diabetes mellitus and depression. $^{34-36}$

Second, at the level of the individual professionals, GPs indicated that there is uncertainty about the additional benefit of PatientCoach in terms of time investment related to improved outcomes in asthma care, as in contrast with usual routine care. GPs are willing to invest if outcomes are favourable for PatientCoach. Not all GPs experience a high sense of urgency towards asthma care. Among some GPs, a more or less passive approach towards asthma care was demonstrated. This seems to be in contrast with work routines of practice nurses, even though not explicitly explored. Indeed, nurses are known to have a proactive approach towards patients with chronic diseases, ${ }^{37}$ thereby providing the type of care required for guiding patients in conducting self-management activities. ${ }^{38}$ The lack of structured asthma care observed within this study has been described in previous literature. ${ }^{7} 3940$ Those practice nurses working within practices without structured asthma care identified a low level of perceived selfefficacy towards asthma care. Even though we did not explicitly analyse which practices were successful in delivering high-quality asthma care, our data suggest that explicit working procedures between GPs and practice nurses are of importance towards achieving this. This corresponds with findings previously described by Wiener-Ogilvie et $a l^{41}$

Third, at the level of the individual patient, not all patients expect a benefit of using PatientCoach in terms of symptom reduction. Patients and professionals found that PatientCoach might not be suitable for those with insufficient control of symptoms, elderly or those with language difficulties. The lack of asthma control has previously been related to willingness to use and outcomes of self-management. ${ }^{42}{ }^{43}$ Research, on asthma action plans-which are an essential part for self-management -indicate that this could lead to offering novel tools like PatientCoach to a very select population group. ${ }^{44}$ Recent studies demonstrated that internet-based tools could improve the clinical outcomes in the elderly population and those with a low socioeconomic status. ${ }^{45} 46$ GPs and practice nurses identified patients with asthma as a difficult target group (ie, lack of medication adherence), which corresponds with current literature. ${ }^{47}$ Patients themselves identified difficulties with changing routines as a barrier, for instance, to take medication regularly or to monitor symptoms regularly. Like professionals, some patients found PatientCoach impersonal. 
Fourth, at the domain of the organisation, practice nurses, in particular, identified a lack of structured asthma care as a barrier. This variation in structured asthma care was also identified among focus groups and inteviews with patients. Other barriers within this domain included availability of staff. ${ }^{39} 40{ }^{48}$ Fifth, at the level of the economic context, a user fee for PatientCoach usage is perceived as a barrier among patients. General practices within the Netherlands are currently not reimbursed for consultations on (internet-based) self-management. This is important as PatientCoach requires an instruction visit that could last 20-30 min. Indeed, sufficient financial resources are a known factor for sustained patientcentred care by using information technology. ${ }^{49}$ Finally, at the domain of the social context, practice nurses identified a lack of support with other practice nurses or GPs within their practices as a barrier. However, practice nurses working in larger practices indicated that they felt supported by colleagues.

\section{Strengths and limitations}

Our study includes several limitations. Our sample was obtained within the province of South Holland. Future research might include a broader geographical area. Another limitation is that, at the time of this study, only a prototype of PatientCoach was available and participants had no experience in using PatientCoach. Therefore, our data are based on their expectations towards PatientCoach usage. Additional insight would be gained from actual user experiences among all participant groups. Currently, the internet is most often accessed by a mobile phone or tablet. ${ }^{50}$ IBSM support should therefore be available for these devices. In spite of these limitations, our study provides in-depth information on barriers to PatientCoach usage, which could be relevant for using internet-based technology in other chronic diseases. Our sample was diverse in terms of variety of practice settings, participant age, level of symptom severity and educational level among patients, level of experience among professionals and educational level of patients. The practice nurses were all female, which reflects this professional population.

\section{Conclusion}

This study provides insight into barriers on implementation of IBSM support as provided by PatientCoach among patients, GPs and practice nurses. Insight into barriers is essential for the development of successful implementation strategies for IBSM support in current care. Future research should be focused on assessing the (cost-) effectiveness of implementation strategies in reallife settings.

Acknowledgements The authors thank all the patients, GPs and practice nurses who participated in this study. They also thank Mirjam Garvelink and Céline van Lint who assisted in conducting focus groups.

Contributors JLVG, MJB, LvB-V, JBS-S and JKS were involved in the design of the study. JLVG moderated the Focus Groups (FGs) and Interviews (IVs). MJB and LvB-V observed the FGs. JLvG performed the transcriptions. Coding was conducted by JLvG and MJB. JLvG drafted the manuscript, which was critically reviewed by all authors. The manuscript has been read and approved by all authors.

Funding This work was supported by grants from the Netherlands Organization for Health Research and Development (ZonMW) (award number 80-82315-97-10004) and the Lung Foundation Netherlands (award number 3.4.09.011). Funding for this publication will be obtained from the Netherlands Organization for Scientific Research (NWO) Incentive Fund Open Access publications.

Competing interests JKS received unrestricted research grants from the Lung Foundation Netherlands, the Netherlands Organisation for Health Research and Development (ZonMW), Fonds NutsOhra, Chiesi NL and GlaxoSmithkline NL.

Ethics approval Medical Ethical Committee of the Leiden University Medical Centre. An exception was obtained, as ethical approval for this type of study is not required under Dutch law (project ID 10.048).

Provenance and peer review Not commissioned; externally peer reviewed.

Data sharing statement All transcripts of interviews and focus groups are available in Dutch. These can be obtained by approaching the corresponding author. Apart from the transcripts, no additional data are available.

Open Access This is an Open Access article distributed in accordance with the Creative Commons Attribution Non Commercial (CC BY-NC 4.0) license, which permits others to distribute, remix, adapt, build upon this work noncommercially, and license their derivative works on different terms, provided the original work is properly cited and the use is non-commercial. See: http:// creativecommons.org/licenses/by-nc/4.0/

\section{REFERENCES}

1. Global Initiative for Asthma (GINA). 2011. Global strategy for asthma management and prevention. 2015. http://www.ginasthma.org/ (accessed 15 Mar 2016).

2. Reddel HK, Jenkins CR, Partridge MR Self-management support and other alternatives to reduce the burden of asthma and chronic obstructive pulmonary disease. Int J Tuberc Lung Dis 2014;18:1396-406.

3. Gibson PG, Powell H, Coughlan J, et al. Self-management education and regular practitioner review for adults with asthma. Cochrane Database Syst Rev 2003:CD001117.

4. Plaza $\mathrm{V}$, Peiró $\mathrm{M}$, Torrejón $\mathrm{M}$, et al. A repeated short educational intervention improves asthma control and quality of life. Eur Respir $J$ 2015;46:1298-307.

5. Steppuhn H, Langen U, Mueters S, et al. Asthma management practices in adults-findings from the German Health Update (GEDA) 2010 and the German National Health Interview and Examination Survey (DEGS1) 2008-2011. J Asthma 2016;53:50-61.

6. Sulaiman N, Aroni R, Thien F, et al. Written Asthma Action Plans (WAAPs) in Melbourne general practices: a sequential mixed methods study. Prim Care Respir J 2011;20:161-9, 1 p following 169.

7. Worth $A$, Pinnock $H$, Fletcher $M$, et al. Systems for the management of respiratory disease in primary care-an international series: United Kingdom. Prim Care Respir J 2011;20:23-32.

8. Morrison D, Wyke S, Agur K, et al. Digital asthma self-management interventions: a systematic review. J Med Internet Res 2014;16:e51.

9. Huckvale K, Car M, Morrison C, et al. Apps for asthma self-management: a systematic assessment of content and tools. BMC Med 2012;10:144

10. van der Meer V, Bakker MJ, van den Hout WB, et al. Internet-based self-management plus education compared with usual care in asthma: a randomized trial. Ann Intern Med 2009;151:110-20.

11. van der Meer V, van Stel HF, Detmar SB, et al. Internet-based self-management offers an opportunity to achieve better asthma control in adolescents. Chest 2007;132:112-9.

12. Wagner $\mathrm{EH}$, Austin BT, Davis $\mathrm{C}$, et al. Improving chronic illness care: translating evidence into action. Health Aff (Millwood) 2001;20:64-78.

13. Thomas M, Kay S, Pike J, et al. The Asthma Control Test (ACT) as a predictor of GINA guideline-defined asthma control: analysis of a multinational cross-sectional survey. Prim Care Respir J 2009;18:41-9.

14. van der Meer V, van den Hout WB, Bakker MJ, et al. Cost-effectiveness of Internet-based self-management compared with usual care in asthma. PLoS ONE 2011;6:e27108. 
15. van Gaalen JL, Beerthuizen T, van der Meer V, et al. Long-term outcomes of internet-based self-management support in adults with asthma: randomized controlled trial. J Med Internet Res 2013;15: e188.

16. Cabana MD, Rand CS, Powe NR, et al. Why don't physicians follow clinical practice guidelines? A framework for improvement. JAMA 1999;282:1458-65.

17. Grol R, Wensing M. What drives change? Barriers to and incentives for achieving evidence-based practice. Med J Aust 2004;180(6 Suppl):S57-60.

18. Pinnock $\mathrm{H}$, Slack $\mathrm{R}$, Pagliari $\mathrm{C}$, et al. Understanding the potential role of mobile phone-based monitoring on asthma self-management: qualitative study. Clin Exp Allergy 2007;37:794-802.

19. Pope C, van Royen P, Baker R. Qualitative methods in research on healthcare quality. Qual Saf Health Care 2002;11:148-52.

20. Geijer RM, Tuut MK, in't Veen JC, et al. (The NHG guidelines 'Adult asthma' and 'COPD']. Ned Tijdschr Geneeskd 2015;159:A9076.

21. CBS: internet gebruik in NL. http://www.cbs.nl/en-GB/menu/themas/ vrije-tijd cultuur/publicaties/artikelen/archief/2015/ tablet-verdringt-bord-van-schoot.htm (accessed 15 Mar 2016).

22. Morgan D, Krueger R, King JA. The Focus Group Guide books. Vol. 1-6. Thousand Oaks, CA: Sage Publications, 1998.

23. Kuper A, Lingard L, Levinson W. Critically appraising qualitative research. BMJ 2008;337:a1035.

24. Juniper EF. Assessing asthma control. Curr Allergy Asthma Rep 2007;7:390-4.

25. Juniper EF, Bousquet J, Abetz L, et al. Identifying 'well-controlled' and 'not well-controlled' asthma using the Asthma Control Questionnaire. Respir Med 2006;100:616-2.

26. Hsieh HF, Shannon SE. Three approaches to qualitative content analysis. Qual Health Res 2005:15:1277-88.

27. Tong A, Sainsbury P, Craig J. Consolidated criteria for reporting qualitative research (COREQ): a 32 -item checklist for interviews and focus groups. Int J Qual Health Care 2007:19:349-57.

28. Mirkovic J, Kaufman DR, Ruland CM. Supporting cancer patients in illness management: usability evaluation of a mobile app. JMIR mHealth uHealth 2014;2:e33.

29. Reynoldson $\mathrm{C}$, Stones $\mathrm{C}$, Allsop $\mathrm{M}$, et al. Assessing the quality and usability of smartphone apps for pain self-management. Pain Med 2014:15:898-909.

30. Saleem JJ, Plew WR, Speir RC, et al. Understanding barriers and facilitators to the use of Clinical Information Systems for intensive care units and Anesthesia Record Keeping: a rapid ethnography. Int J Med Inform 2015;84:500-11.

31. Bagozzi RPD, F.D. Development and Test of a Theory of Technological Learning and Usage (abstract). Human Relations. http://hum.sagepub.com/content/45/7/659.abstract

32. Scandurra I, Hägglund M, Koch S. From user needs to system specifications: multi-disciplinary thematic seminars as a collaborative design method for development of health information systems. $J$ Biomed Inform 2008;41:557-69.

33. Van Velsen L, Wentzel J, Van Gemert-Pijnen JE. Designing eHealth that Matters via a Multidisciplinary Requirements Development Approach. JMIR Res Protoc 2013;2:e21.
34. Dwarswaard J, Bakker EJ, van Staa A, et al. Self-management support from the perspective of patients with a chronic condition: a thematic synthesis of qualitative studies. Health Expect 2016;19:194-208.

35. Edwards L, Thomas C, Gregory A, et al. Are people with chronic diseases interested in using telehealth? A cross-sectional postal survey. J Med Internet Res 2014:16:e123.

36. Vassilev I, Rowsell A, Pope C, et al. Assessing the implementability of telehealth interventions for self-management support: a realist review. Implement Sci 2015;10:59.

37. Seale C, Anderson E, Kinnersley P. Treatment advice in primary care: a comparative study of nurse practitioners and general practitioners. J Adv Nurs 2006:54:534-41.

38. Newcomb PA, McGrath KW, Covington JK, et al. Barriers to patient-clinician collaboration in asthma management: the patient experience. J Asthma 2010;47:192-7.

39. Griffiths P, Maben J, Murrells T. Organisational quality, nurse staffing and the quality of chronic disease management in primary care: observational study using routinely collected data. Int J Nurs Stud 2011;48:1199-210.

40. Newbould J, Burt $\mathrm{J}$, Bower $\mathrm{P}$, et al. Experiences of care planning in England: interviews with patients with long term conditions. $B M C$ Fam Pract 2012;13:71.

41. Wiener-Ogilvie S, Pinnock H, Huby G, et al. Do practices comply with key recommendations of the British Asthma Guideline? If not, why not? Prim Care Respir J 2007:16:369-77.

42. Thoonen BP, Schermer TR, Van Den Boom G, et al. Self-management of asthma in general practice, asthma control and quality of life: a randomised controlled trial. Thorax 2003:58:30-6.

43. van der Meer V, van Stel HF, Bakker MJ, et al. Weekly self-monitoring and treatment adjustment benefit patients with partly controlled and uncontrolled asthma: an analysis of the SMASHING study. Respir Res 2010;11:74.

44. Ring N, Jepson R, Hoskins G, et al. Understanding what helps or hinders asthma action plan use: a systematic review and synthesis of the qualitative literature. Patient Educ Couns 2011;85:e131-43.

45. Aalbers T, Baars MA, Rikkert MG. Characteristics of effective Internet-mediated interventions to change lifestyle in people aged 50 and older: a systematic review. Ageing Res Rev 2011;10:487-97.

46. Brown J, Michie S, Geraghty AW, et al. Internet-based intervention for smoking cessation (StopAdvisor) in people with low and high socioeconomic status: a randomised controlled trial. Lancet Respir Med 2014;2:997-1006.

47. Goeman DP, Hogan CD, Aroni RA, et al. Barriers to delivering asthma care: a qualitative study of general practitioners. Med J Aust 2005; 183:457-60

48. Loignon C, Bedos C, Sevigny R, et al. Understanding the self-care strategies of patients with asthma. Patient Educ Couns 2009;75:256-62

49. Finkelstein J, Knight A, Marinopoulos S, et al. Enabling patient-centered care through health information technology. Evid Rep Technol Assess (Full Rep). 2012;206:1-1531.

50. CBS (Bureau Statistics): meer internet gebruik via mobiel dan pc. http://www.cbs.nl/en-GB/menu/themas/dossiers/eu/publicaties/ archief/2013/2013-3851-wm.htm (accessed 15 Mar 2016). 